\section{Katheterset erhält Designpreis}

— Das Katheterset SpeediCath ${ }^{\oplus}$ Compact Set wurde mit dem red dot design award ausgezeichnet, ein Preis für Produktdesign, der seit 1955 jährlich vergeben wird und bei dem die Anwenderfreundlichkeit und Ästhetik der Produkte im Fokus stehen. „Coloplast setzt neue Maßstäbe in der Kathetergestaltung", lautete die Botschaft der Jury, die das Katheterset als ikonisch, klar und nicht stigmatisierend beschrieb.

Bei dem SpeediCath ${ }^{\circledast}$ Compact Set handelt es sich um einen kompakten Katheter mit integriertem sterilen Urinbeutel. Dank seiner einzigartigen Beschichtung ist der Einmalkatheter sofort und überall gebrauchsfertig. "Natürlich ist es für jeden Designer eine Ehre, wenn sein Design gewürdigt wird", sagte Marlene Corydon, Senior Project Manager bei Coloplast. Verglichen mit herkömmlichen Kathetern, die in der Öffentlichkeit leicht unerwünschte Aufmerksamkeit auf sich ziehen können, ist das SpeediCath ${ }^{\circledR}$ Compact Set ein diskretes, nicht stigmatisierendes Hilfsmittel. „Jetzt wird nicht jeder zufällige Blick in die Handtasche gleich zu einer peinlichen Situation“, erklärte Corydon.

Das Designteam des SpeediCath ${ }^{\oplus}$ Compact Set wird die Auszeichnung im Juli 2013 entgegennehmen. Die ausgezeichneten Produkte werden im red dot design museum in Essen ausgestellt.

Nach Informationen von Coloplast, Hamburg

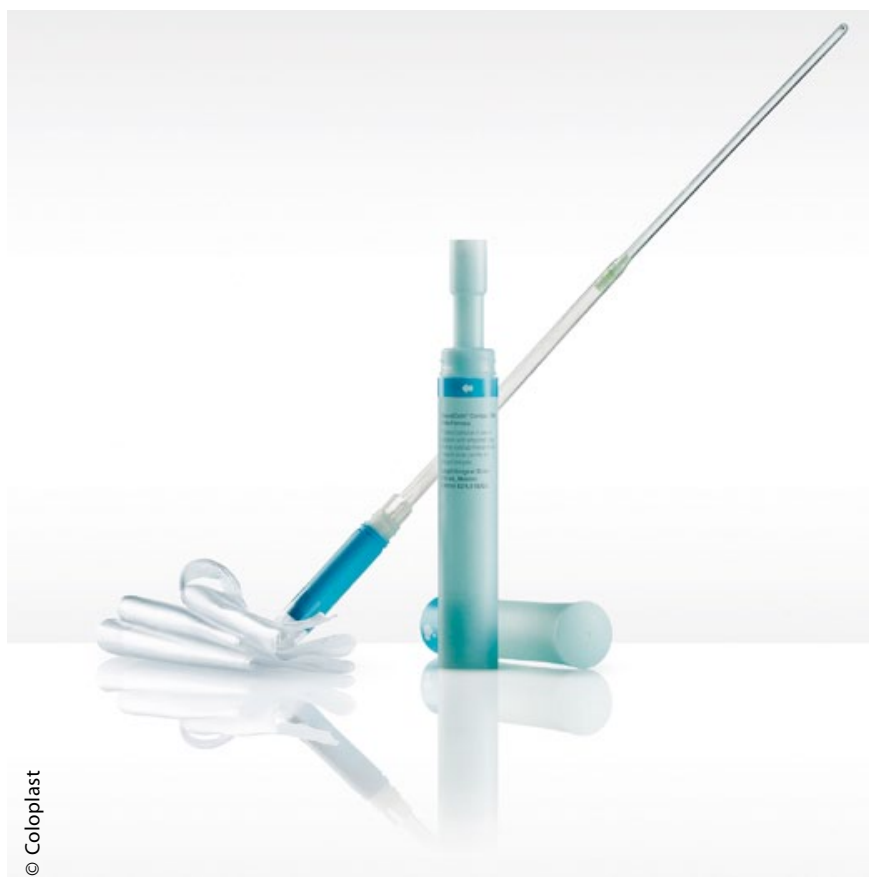

Praktisch für unterwegs: Der kompakte Katheter mit integriertem Beutel ist klein, diskret und sofort gebrauchsfertig.

\section{Wundheilung mit Schafgarbe und Echter Kamille}

— Die antiphlogistischen, spasmolytischen und karminativen Wirkungen der Echten Kamille sind neben den bakteriostatischen und fungistatischen Effekten wissenschaftlich belegt. „In den letzten Jahren sind für weitere wichtige Bestandteile der ätherischen Öle der Kamille wie Apigenin, Bisabolol, Chamazulen und den Flavonen therapeutische wundheilende Wirkungen bekannt geworden. Insbesondere die Ulkusprotektion und Granulationsförderung sowie die Anregung des Hautstoffwechsels", erklärte Prof. Uwe Wollina, Dresden-Friedrichstadt.

Auch die Schafgarbe wirkt entzündungshemmend. „Das Besondere der Schafgarbe sind jedoch ihre Gerbstoffe, die Tannine. Sie schließen mikroskopische Verletzungen, um die Intaktheit der Hautstruktur wiederherzustellen. Dadurch wirken sie an der Oberfläche der Haut blutstillend, bauen einen schützenden Film auf und beugen weiteren Reizungen vor", so Wollina.

Pharma Wernigerode Kamillan ${ }^{\circledR}$ vereint die positiven Synergien von Kamille und Schafgarbe und beschleunigt so den Heilungsprozess bei Entzündungen von Haut und Schleimhaut, zum Beispiel als Zusatz von Teil- und Sitzbädern bei Entzündungen im sensiblen Genital- und Analbereich.

Nach Informationen von Aristo Pharma, Berlin

\section{Erstes generisches Sildenafil auf dem Markt}

— Seit dem 1. Juni steht mit Sildenafil Pfizer eine generische Version des Originalpräparats Viagra ${ }^{\circledast}$ in der Originalgalenik zur Verfügung. Sildenafil Pfizer wird in drei verschiedenen Dosierungen ( $25 \mathrm{mg}, 50 \mathrm{mg}$ und $100 \mathrm{mg}$ ) und Packungsgrößen (4, 12 und 24 Tabletten) angeboten. Sildenafil Pfizer unterscheidet sich vom Originalpräparat durch die Verpackung und Tablettenfarbe - die rautenförmigen Tabletten sind weiß statt blau. Auch Sildenafil Pfizer ist ein verschreibungspflichtiges Medikament und muss in einer Apotheke oder zertifizierten Online-Apotheke erworben werden. Viagra ${ }^{\circledR}$ ist auch nach dem Patentablauf Ende Juni in bewährter Form erhältlich.

Sildenafil wirkt durch eine hochpotente und hochselektive Hemmung des Enzyms Phosphodiesterase-5 (PDE-5) und verstärkt den Blutfluss in den Penis. Dadurch hilft das Medikament Männern, eine hohe Erektionsqualität mit einer entsprechenden Erektionshärte zu erreichen und die Erektion aufrechtzuerhalten. Es war das erste Präparat dieser Wirkstoffklasse. Da mit der Zunahme typischer Grunderkrankungen wie Diabetes mellitus und Bluthochdruck auch die Prävalenz der Erektilen Dysfunktion steigen wird, ist der Bedarf an zusätzlichen Behandlungsoptionen groß.

Nach Informationen von Pfizer, Berlin 